\title{
Políticas públicas e o desenvolvimento sustentável de cidades litorâneas: estudo de caso Bombinhas (SC) e Ilhabela (SP)
}

Nádia Mara Franz

nadiamfranz@gmail.com

Paraná - UTFPR, Curitiba, Paraná, Brasil

Cleverson Vitorio Andreoli

cleverson@andreoliambiental.com.br Instituto Superior de Administração e Economia, Curitiba, Paraná, Brasil

Christian Luiz da Silva christiansilva@utfpr.edu.br Universidade Tecnológica Federa do Paraná - UTFPR, Curitiba, Paraná, Brasil

\section{RESUMO}

A pesquisa apresenta estudos de caso e tem como objetivo identificar políticas que visam estimular o desenvolvimento sustentável nas cidades turística litorâneas de Bombinhas e Ilhabela. Fundamentado em conceitos sobre desenvolvimento sustentável, o estudo identifica e descreve ações e políticas adotadas pela administração pública nos pilares do desenvolvimento sustentável e analisa os efeitos utilizando indicadores de desempenho. A pesquisa tem as características da abordagem mista, de natureza aplicada, com delimitação e design técnico de estudo de casos múltiplos. As cidades avaliadas obtiveram crescimento de população, PIB e PIB per capita no período mensurado. Entretanto, o crescimento da renda não se refletiu na mesma proporção nos indicadores sociais e ambientais. $O$ crescimento econômico pode ser o meio de gerar o progresso social e o ambiente saudável, porém a maximização dos seus reflexos na sustentabilidade depende das escolhas que os gestores públicos e a sociedade fazem para o futuro de suas cidades.
\end{abstract}

PALAVRAS-CHAVE: Desenvolvimento Sustentável, Políticas Públicas; Indicadores de Desempenho. 


\section{INTRODUÇÃO}

As administrações públicas municipais brasileiras ganharam maior autonomia administrativa e a distribuição das verbas públicas foi ampliada com a Constituição de 1988, entretanto foram estabelecidos limites rígidos na aplicação dos recursos e seus atos são fiscalizados pelos Tribunais de Contas da União. Por outro lado, a população das áreas urbanas cresce a cada ano e cerca de $86 \%$ da população brasileira já vive em áreas urbanas. Esse cenário desafia os gestores públicos a buscar soluções inovadoras e sustentáveis. que promovam o crescimento econômico com benefícios sociais e ambientais. O turismo, que já representa 3,7\% do PIB brasileiro, aponta como uma das formas viáveis ao crescimento econômico das cidades, mas também passa pelo mesmo desafio de ser sustentável.

Neste contexto que reside o interesse e relevância da pesquisa, de saber se é possível termos uma administração pública municipal alinhada às práticas de governança e de gestão participativa que estimule o desenvolvimento sustentável em suas três vertentes: econômico, social e ambiental.

O referencial teórico busca fundamentar a coleta e a análise dos dados nos conceitos de desenvolvimento sustentável, detalhando o Objetivo de Desenvolvimento Sustentável (ODS) 11.

As cidades litorâneas pesquisadas Ilhabela/SP e Bombinhas/SC destacaram-se no ranking nacional do IGM - Índice de Governança Municipal 2017 do CFA Conselho Federal de Administração onde ocuparam o primeiro e terceiro lugar respectivamente. $O$ índice avaliou três dimensões: finanças, gestão e desempenho.

O objetivo da pesquisa é identificar políticas públicas que visam promover o desenvolvimento sustentável pelas administrações públicas da cidade litorânea de Bombinhas/SC e Ilhabela/SP. Para tal, o estudo identifica e descreve ações e políticas adotadas pela administração pública municipal nos três pilares do desenvolvimento sustentável: econômico, social e ambiental; e analisa os resultados utilizando indicadores de desempenho específicos, relacionados a estas dimensões.

O trabalho tem a abordagem mista de pesquisa qualitativa e quantitativa, de natureza aplicada, com delimitação e design técnico de estudo de caso. $O$ estudo está estruturado em cinco seções: introdução que enfatiza a relevância e o objetivo do estudo, referencial teórico, procedimentos metodológicos, análise e discussão dos resultados e considerações finais.

\section{REFERENCIAL TEÓRICO}

\subsection{Desenvolvimento sustentável e as cidades}

Segundo dados das Nações Unidas (2018), mais de 50\% da população mundial habita nas cidades e até 2030 deverá chegar à $70 \%$, sendo que destes, 10\% vivem em assentamentos precários e deve triplicar até 2050, ou seja, chegar a três bilhões de pessoas. Na América Latina $75 \%$ da população vivem em áreas urbanas e no Brasil este índice ultrapassa a $86 \%$. 
Contudo, as cidades carecem de infraestrutura para abrigar tamanha população, e há o desencadeamento de uma série de problemas como falta de saneamento, moradia, acesso à saúde e educação. A Organização das Nações Unidas (ONU) alerta que as cidades consomem mais de $75 \%$ do total de energia produzida e emitem $80 \%$ dos gases que causam o aquecimento global. A fome, desemprego, desigualdade social, mobilidade caótica e degradação do meio ambiente se somam aos desafios a serem repensados pelos gestores públicos e sociedade a fim de que as cidades encontrem o caminho para a sustentabilidade.

"O conceito de cidades sustentáveis requer uma nova lógica de funcionamento, gestão e crescimento em detrimento das que foram praticadas no século XX, seguindo a ideia de crescimento com esgotamento". (Leite, 2012, apud Rego, et.al. 2013, p. 549).

Sachs $(2009$, p. 53) ressalta que é necessário adotar padrões negociados e contratuais de gestão de biodiversidade, estabelecer o uso racional e sustentável dos recursos naturais em prol das populações locais e incorporar a conservação da biodiversidade como um componente de estratégia de desenvolvimento.

Diante desta realidade, percebe-se que temas que envolvem as cidades têm tomado a agenda de órgãos governamentais e não-governamentais, nacionais e internacionais, que se debruçam em estudos para transformar o tratado global dos Objetivos de Desenvolvimento Sustentável - ODS em ações locais nas cidades, na área rural e nos assentamentos, pois é nestes espaços que permeiam a agenda do desenvolvimento e o alcance dos ODS até 2030.

O ODS 11 que prevê tornar as cidades e os assentamentos humanos inclusivos, seguros, resilientes e sustentáveis, se relaciona ao desenvolvimento sustentável das cidades e para ser alcançado é necessário que se avance no cumprimento das metas dos outros objetivos, dado sua interdependência com os demais ODS, pois entre suas metas estão o acesso a todos de habitação com energia, saneamento básico, transporte e segurança, buscando a eficiência dos recursos. O ODS 11 apoia planejamentos urbanos integrados ao peri urbano e rural que promovam o crescimento econômico, o bem-estar social e recuperem e protejam o meio ambiente, mitigando as mudanças climáticas, as catástrofes e as mortes decorrentes destas.

De acordo com a ONU (2018), os planejamentos urbanos devem contemplar soluções de conflitos já existentes como a pobreza e a desigualdade, atuar na prevenção e adaptação às mudanças climáticas e ser um agente transformador aliado na inovação.

"Toda cidade sustentável se desenvolve a partir de uma ligação adequada, respeitada e ponderada entre o meio ambiente construído e a geografia natural. Portanto, planejar todas as etapas da urbanização é essencial para que a cidade possa ser bem cuidada". (...) "Sendo assim, são necessários modelos contemporâneos adequados ao desenvolvimento sustentável, reformulados conjuntamente, pela atuação pública e privada". (LEITE, 2012 apud REGO, et. al. 2013, pp. 548 - 549).

Segundo o Programa Cidades Sustentáveis (2012), as cidades sustentáveis caracterizam-se por boa gestão e governança pública, planejamento, consciência política, participação social, economia responsável, compromisso com os valores humanos, cuidado com a natureza e as pessoas, ação local e visão global. 


\section{METODOLOGIA}

O trabalho tem a abordagem mista, de natureza aplicada, com delimitação e design técnico de estudo de caso e realista do ponto de vista epistemológico. 0 objetivo é de caráter exploratório descritivo e analítico. Os procedimentos técnicos para a coleta de dados são a pesquisa documental, a entrevista semiestruturada e a observação não participante. A análise dos dados é conduzida pela estratégia analítica fundamentada em referencial teórico. (SAMPIERI et.al. 2013 e YIN, 2015).

A coleta de dados inclui consultas à documentos como plano diretor, leis, decretos e planos plurianuais. Para tal, a fonte de dados utilizada para o estudo é de origem secundária. As entrevistas semiestruturadas ao gestor público segue um roteiro de temas que tem a finalidade de compreender com maior profundidade $o$ conteúdo dos documentos, obter pontos de vista diferentes sobre determinado tema e conseguir outras informações além das encontradas nos documentos. 0 pesquisador visita à cidade, para observação da cultura, da percepção do povo sobre as questões elencadas no estudo, para validar a análise dos documentos e entrevistas, bem como explorar aspectos pouco comuns ou não encontrados na pesquisa documental.

Os dados quantitativos coletados são numéricos e representam indicadores de desempenho da cidade. os indicadores utilizados, relacionados no quadro 3 , foram referenciados às dimensões ambiental, social e econômica. As coletas dos dados são obtidas de acordo com uma série temporal em sites oficiais públicos do IBGE - Instituto Brasileiro de Geografia e Estatística, SNIS - Sistema Nacional de Informações sobre Saneamento, IPEA - Instituto de Pesquisa Econômica Aplicada, DATASUS - Departamento de Informática do Sistema Único de Saúde, ANEEL Agência Nacional de Energia Elétrica e INEP - Instituto Nacional de Estudos e Pesquisas Educacionais. O software Minitab V. 18 é empregado como ferramenta computacional para gerar os gráficos da evolução temporal das variáveis. A partir destes gráficos é elaborado quadro resumo da evolução das variáveis.

A análise e apresentação dos dados utiliza a estratégia de embasamento em proposições teóricas, pois há estudos sobre o desenvolvimento sustentável de cidades turísticas que orienta a condução, organização e análise dos dados.

\section{ANÁLISE E DISCUSSÃO DOS RESULTADOS}

Para compreender como se busca promover o desenvolvimento sustentável pelas administrações públicas das cidades litorâneas de Bombinhas/SC e Ilhabela/SP é primordial conhecer o ambiente dessas cidades, para na sequência identificar as políticas atuais em destaque que buscam promover o desenvolvimento sustentável e por fim examinar os indicadores de desempenho.

As cidades pesquisadas espelham a rica diversidade presente nos municípios brasileiros seja por fatores históricos, geográficos, demográficos ou culturais. A Tabela 1 - Indicadores de perfil dos municípios resume as características do ambiente dessas cidades. 
Tabela 1 - Indicadores de Perfil dos Municípios

\begin{tabular}{|c|c|c|}
\hline Perfil\Cidade & Bombinhas/SC & Ilhabela/SP \\
\hline População estimada 2017 & 18.623 & 33.354 \\
\hline Área km2 & 35,923 & 346,389 \\
\hline Unidade de Conservação & $\begin{array}{c}\text { Morro do Macaco, Galheta, } \\
\text { Reserva do Arvoredo e a } \\
\text { Costeira de Zimbros (19,5 km2) }\end{array}$ & $\begin{array}{l}\text { Parque Estadual de } \\
\text { Ilhabela }(270 \mathrm{~km} 2)\end{array}$ \\
\hline Economia (tipo) & Turismo e Pesca (Mergulho) & $\begin{array}{c}\text { Turismo e Comércio. } \\
\text { (Vela) }\end{array}$ \\
\hline Energia limpa ou reuso da água & $\begin{array}{l}\text { Captação da água da } \\
\text { chuva/prédios }\end{array}$ & Energia solar/Boneto \\
\hline Mobilidade & $\begin{array}{l}\text { Transporte coletivo não } \\
\text { integrado }\end{array}$ & $\begin{array}{c}\text { Balsa, Transporte coletivo } \\
\text { não integrado }\end{array}$ \\
\hline Plano Diretor e Plurianuais & Sim & Sim \\
\hline Portal da Transparência & Sim & Sim \\
\hline Internet pública & Em implantação & Sim \\
\hline
\end{tabular}

Fonte: Elaboração própria (2018).

Observa-se na Tabela 1 que os municípios, mesmo sendo em regiões distantes do Brasil, com cultura e geografia variadas, apresentam dados que revelam pontos importantes em comum a serem analisados. Percebe-se que as cidades têm suas economias baseadas no turismo diretamente relacionado às suas belezas e recursos naturais, possuem expressivas áreas ambientais protegidas, porém a mobilidade em certas cidades apresenta dificuldades e as iniciativas de energia limpa e reuso dos recursos naturais são incipientes.

Verifica-se que há instrumentos de controle social implantados e que o uso da tecnologia tem se tornado um aliado às administrações públicas no relacionamento com à sociedade. Oliveira e Rezende (2017) destacam o uso das TICs como ferramenta vital para a gestão estratégica e de desempenho dos governos municipais, auxiliando as cidades em sua transformação para cidades inteligentes e sustentáveis.

Segundo Castellà e Parés (2012), os municípios desenvolvem experiências das mais diversas de democracia participativa com o propósito de administrar sociedades cada vez mais diversificadas e complexas. Considerando a territorialidade, o capital social das cidades, o estudo identifica e descreve as políticas públicas que buscam promover o desenvolvimento sustentável. A Tabela 2 apresenta o resumo das políticas que se destacaram na coleta de dados. 
Tabela 2 - Ações e Políticas relacionadas ao desenvolvimento sustentável

\begin{tabular}{|r|l|}
\hline Econômica & \\
\hline Bombinhas/SC & Projeto Orla - Projeto de Gestão Integrada da Orla Marítima \\
\hline Social & $\begin{array}{l}\text { Projeto Orla - Projeto de Gestão Integrada da Orla Marítima } \\
\text { Agenda Anual de Eventos para a Geração de Renda }\end{array}$ \\
\hline Bombinhas/SC & $\begin{array}{l}\text { Garantia de atendimento de 100\% da saúde básica } \\
\text { PM de Educação de Bombinhas 2015 - 2025. Educação Integral }\end{array}$ \\
\hline Ilhabela/SP & $\begin{array}{l}\text { Regularização Fundiária } \\
\text { Fundação da Casa dos Conselhos }\end{array}$ \\
\hline Ambiental & $\begin{array}{l}\text { Expansão do saneamento básico para 97\% do município até } 2021 \\
\text { Retomada da Coleta seletiva de lixo e projeto Bota Fora. }\end{array}$ \\
\hline Ilhabela/SP & \begin{tabular}{l} 
Expansão do Saneamento Básico aos Bairros \\
\hline
\end{tabular} \\
\hline
\end{tabular}

Quando da coleta de dados sobre a tríade econômica-social-ambiental, observa-se a polarização inicial das prioridades da sociedade, que se concentram na geração de renda e preservação do meio ambiente, especialmente com a questão do esgoto sanitário. As questões que envolvem o saneamento básico nessas cidades estão mais evidentes à sociedade, pois elas dependem economicamente, pelo turismo, de suas belezas e recursos naturais, que estão sendo comprometidas pela falta de saneamento básico adequado, e não pela causa de outros problemas, como à saúde e evasão escolar, por exemplo. Os temas prioritários relacionados à dimensão social se concentram na saúde e educação, e a maioria das pessoas tem o entendimento que a melhoria desses depende diretamente do crescimento econômico e não das escolhas na priorização das políticas públicas do município.

Observa-se que as cidades onde as políticas públicas integram no seu processo a participação social, também demonstram o exercício dos princípios da governança pública de legitimidade, equidade, responsabilidade, eficiência, probidade, transparência e prestação de contas (accountalility). Considera-se, portanto, que uma gestão pública participativa coexiste com os princípios de governança pública.

Ademais, cada cidade apresenta um nível de amadurecimento distinto no processo de gestão participativa e nas práticas de governança pública. $\mathrm{O}$ nível de maturidade dos processos de políticas públicas analisados apresenta relação com a história e cultura da cidade, ou seja, com sua territorialidade.

A participação social na elaboração e controle das políticas públicas têm alterado a prioridade das implementações das políticas públicas e a forma de relacionamento entre a sociedade e a administração pública municipal. Quanto mais organizada e atuante for a sociedade civil na gestão do seu munícipio, mais esta sociedade tem o sentimento de pertencimento e comprometimento com o futuro de sua cidade.

Segundo Mitchell e Fazi (2017), é possível mudar o status quo, com uma visão progressista e emancipadora da soberania nacional, baseada na soberania 
popular, no controle democrático da economia, no pleno emprego, na justiça social, na redistribuição dos ricos para os pobres, na inclusão e na transformação sócio ecológica da produção e da sociedade. A soberania popular é alcançada pela participação social no processo das políticas públicas.

Contudo, para concluir a análise dos efeitos das políticas aplicadas no desenvolvimento sustentável de cidades sob administrações que conduzem as suas gestões de formas participativa e alinhadas às práticas de governança pública, total ou parcialmente, são examinados a evolução temporal de indicadores que objetivam avaliar aspectos da sustentabilidade de uma cidade. A Tabela 3 apresenta indicadores selecionados da norma NBR ISO 37120.2017 da Associação Brasileira de Normas Técnicas (ABNT) relacionada ao desenvolvimento sustentável das cidades e resume os resultados das relações positivas, negativas ou oscilantes encontradas na evolução temporal dos indicadores de desempenho efetuados através de gráficos de dispersão.

Tabela 3 - Evolução dos Indicadores de Desempenho

\begin{tabular}{|c|c|c|}
\hline CIDADE/INDICADOR & BOMBINHAS & ILHABELA \\
\hline População (1999 - 2017) & Positiva & Positiva \\
\hline \multicolumn{3}{|l|}{ Econômico } \\
\hline PIB (1999 - 2014) & Positiva & Positiva \\
\hline PIB per capita (1999 - 2014) & Positiva & Positiva \\
\hline IDHM (1991 - 2010) & Positiva & Positiva \\
\hline \multicolumn{3}{|l|}{ Social } \\
\hline Taxa mortalidade infantil (2008 - 2014) & $\begin{array}{l}\text { Oscilante com piora nos } \\
\text { últimos anos }\end{array}$ & $\begin{array}{c}\text { Oscilante com piora nos } \\
\text { últimos anos }\end{array}$ \\
\hline Taxa analfabetismo (1991 - 2010) & Positiva & Positiva \\
\hline IDEB anos iniciais (2005 - 2015) & Positiva & $\begin{array}{l}\text { Oscilante com melhora } \\
\text { nos últimos anos }\end{array}$ \\
\hline IDEB anos finais (2005 - 2015) & $\begin{array}{l}\text { Oscilante com piora nos } \\
\text { últimos anos }\end{array}$ & Positiva \\
\hline Média salários mínimos (2007 - 2015) & $\begin{array}{c}\text { Oscilante com melhora nos } \\
\text { últimos anos }\end{array}$ & $\begin{array}{l}\text { Oscilante com melhora } \\
\text { nos últimos anos }\end{array}$ \\
\hline Taxa homicídios (2003 - 2013) & $\begin{array}{l}\text { Oscilante com melhora nos } \\
\text { últimos anos }\end{array}$ & $\begin{array}{l}\text { Oscilante com melhora } \\
\text { nos últimos anos }\end{array}$ \\
\hline \multicolumn{3}{|l|}{ Ambiental } \\
\hline Abastecimento de água (2006 - 2015) & Positiva & $\begin{array}{c}\text { Oscilante com piora nos } \\
\text { últimos anos }\end{array}$ \\
\hline Esgotamento sanitário (2006 - 2015) & $\begin{array}{l}\text { Sem alteração significativa } \\
\text { - baixo percentual }\end{array}$ & Positiva \\
\hline $\begin{array}{r}\text { Distribuição de energia elétrica (2003 - } \\
\text { 2013) }\end{array}$ & Positiva & Positiva \\
\hline
\end{tabular}


Verifica-se na tabela acima que os indicadores da dimensão econômica e da evolução da população das cidades obtiveram crescimento tanto na população, como no PIB, PIB per capita e IDHM no período mensurado. Na dimensão social a educação demonstra taxas de analfabetismo com melhora no período. A mesma relação percebe-se nas notas do IDEB - anos iniciais, mesmo com oscilações ao longo dos anos. Nas notas do IDEB - anos finais a evolução positiva não é percebida em ambas as cidades. A quantidade média de salários mínimos se manteve estável ao longo período, com tendência de melhora. A taxa de mortalidade infantil e de homicídios apresentam oscilações em todas as cidades no período mensurado. No pilar ambiental, percebe-se a precariedade do saneamento no Brasil, principalmente com relação ao esgotamento sanitário. $\mathrm{Na}$ questão do abastecimento de energia elétrica identifica-se tendência positiva significativa.

Considerando o contexto geral das três dimensões analisadas, a evolução dos indicadores sociais e ambientais analisados não refletiu na mesma proporção do crescimento econômico destas cidades. A análise dos indicadores demonstra que o crescimento econômico por si só, não acarreta melhoria nos benefícios sociais e ambientais. É preciso que haja escolhas políticas alinhadas aos anseios da população, que se obtém pela participação efetiva do cidadão no processo de políticas públicas e em planejamentos e ações com visão de futuro, de médio e longo prazo, como o investimento em saúde, educação e saneamento básico.

Sachs (2007, p. 23) atenta para o fato que o crescimento econômico, por si só não gera o desenvolvimento sustentável, e sim é o instrumento de se obter a inclusão social e o meio ambiente. Segundo o autor é necessário criar espaços nos níveis locais para promover o debate e negociação pelos representantes de todas as forças vivas da sociedade, e que esta pactuação local se transferirá aos níveis regionais e federais. "A visão do desenvolvimento no futuro é o de um desenvolvimento participativo e negociado. Ou seja, deve-se organizar o debate em todos os níveis, desde o desenvolvimento local." (Sachs, 2007, p.28). Desta forma, Sen $(2010$, p.57) corrobora com a conclusão que:

O crescimento econômico pode ajudar não só elevando rendas privadas, mas também possibilitando ao Estado financiar a seguridade social e a intervenção governamental ativa. Portanto, a contribuição do crescimento econômico tem de ser julgada não apenas pelo aumento de rendas privadas, mas também pela expansão dos serviços sociais que o crescimento econômico pode possibilitar.

\section{CONSIDERAÇÕES FINAIS}

Com o objetivo de compreender como se busca fomentar o desenvolvimento sustentável nas cidades litorâneas de Bombinhas/SC e Ilhabela/SP foram identificadas e descritas políticas das administrações públicas da amostra, que utilizam a gestão participativa e os princípios da governança pública na promoção do desenvolvimento sustentável e foram comparados indicadores de desempenho que expressam aspectos importantes da sustentabilidade de uma cidade.

$\mathrm{Na}$ identificação das políticas observou-se a polarização inicial das prioridades da sociedade, que se concentram na geração de renda e preservação do meio ambiente, especialmente com a questão do esgotamento sanitário. As deficiências do saneamento básico, além dos impactos ambientais, afetam diretamente a 
economia do local que depende de suas belezas e recursos naturais para a promoção do turismo e geração de renda.

Os temas prioritários relacionados à dimensão social se concentraram na saúde e educação. Dos princípios da governança pública ficou mais evidente, na coleta de dados, a cobrança da sociedade por transparência e prestação de contas dos gestores públicos, bem como o acesso às informações em linguagem simples e clara.

Na referência dessas ações e políticas com as dimensões da sustentabilidade verificou-se que as mesmas se inter-relacionam e interdependem entre si. Os estudos evidenciaram que gestões públicas participativas exercem, total ou parcialmente, os princípios da governança pública: de legitimidade, equidade, responsabilidade, eficiência, probidade, transparência e prestação de contas (accountability).

Constatou-se que o exercício da participação cidadã e das práticas de governança conduzem ao amadurecimento da sociedade e da gestão pública minimizando os efeitos dos impasses e obstáculos que surgem no decorrer do processo das políticas públicas. Além disso, as etapas de uma política pública, enquanto diagnóstico dos problemas da sociedade, priorização (agenda), formulação, legalização, implementação e avaliação são validadas com a participação social promovidas por gestões públicas participativas

A análise de indicadores que espelham aspectos essenciais do desenvolvimento sustentável de uma cidade confirmou proposições trazidas por autores como Sachs $(2007,2009)$, Sen (2010), que o crescimento econômico é importante, mas é preciso realizar escolhas políticas adequadas para que se transforme em benefícios sociais e ambientais, como em saúde, educação e saneamento básico. A evolução positiva do PIB e PIB per capita não conduz necessariamente ao progresso social e ao meio ambiente saudável. As cidades analisadas, mesmo tendo essas evoluções positivas, ainda se observa que a mortalidade infantil (ambas), o IDEB nos anos finais e saneamento (Bombinhas) e o abastecimento de água (Ilha Bela) não responderam da mesma maneira.

Ademais, o Brasil é um país de território continental onde a diversidade é imensa, seja pela sua história, cultura, geografia ou fatores socioeconômicos, e cada gestão pública em conjunto com a sociedade deve encontrar a melhor forma de incorporar a participação social no processo das políticas públicas locais, a fim de que a população seja ouvida em seus anseios por melhoria na geração da renda, benefícios sociais e ambientais. Os modelos de ferramentas de gestão e de planejamento patrocinados pelo governo federal auxiliam os gestores públicos em suas atribuições, porém precisam ser adequados a territorialidade local.

O controle social aliado às práticas de governança pública na formatação e controle das políticas públicas se apresenta como um caminho possível para alcançar a soberania popular sobre o processo de políticas públicas que promovam o desenvolvimento sustentável das cidades. 


\title{
Public policies and the sustainable development of litoran tourist cities: case study Bombinhas (SC) and Ilhabela (SP)
}

\begin{abstract}
The research presents case studies and aims to identify policies that stimulate sustainable development in the tourist cities of Bombinhas and Ilhabela. Based on concepts of sustainable development, the study describes actions and policies adopted by public administration in the pillars of sustainable development and analyzes the effects using performance indicators. The research has the characteristics of the mixed approach, with delimitation of multiple case studies. The evaluated cities obtained population growth, GDP and per capita GDP in the period measured. However, income growth was not reflected in the same proportion in social and environmental indicators. Economic growth may be the means of generating social progress and a healthy environment but maximizing its impact on sustainability depends on the choices that public managers and society make for the future of their cities.
\end{abstract}

KEY WORDS: Sustainable Development, Public Policies; Performance Indicators. 


\section{REFERÊNCIAS}

ANEEL - Agência Nacional de Energia Elétrica. Acesso em 20 de novembro, 2017. Disponível: http://www.aneel.gov.br.

ABNT - Associação Brasileira de Normas Técnicas. Acesso em 23 de maio, 2018. Disponível: http://abnt.org.br.

Castellà, C. \& Parés, M. (2012). Participação e qualidade democrática: uma proposta de critérios de qualidade. Cidadania e desenvolvimento local: critérios e análise. Rio de Janeiro: Ed. FGV, pp. 207-251.

CFA - Conselho Federal de Administração. Acesso em 16 de junho, 2018.

Disponível: http://igm.cfa.org.br/

DATASUS - Departamento de Informática do sistema único de saúde. Acesso em 10 de novembro, 2017. Disponível:

http://www2.datasus.gov.br/DATASUS/index.php.

IBGE - Instituto Brasileiro de Geografia e Estatística. Acesso de 05 a 16 de dezembro, 2017 e em 10 de maio, 2018. Disponível: https://www.ibge.gov.br .

INEP - Instituto Nacional de Estudos e Pesquisas Educacionais Anísio Teixeira. Acesso em 21 de janeiro, 2018. Disponível: http://ideb.inep.gov.br/resultado .

IPEA - Instituto de Pesquisa e Economia Aplicada. Acesso em 16 de novembro, 2017. Disponível: http://www.ipea.gov.br/portal .

Mitchell, W. \& Fazi, T. (2017). Reclaiming the State. Universidade de Chicago.

Nações Unidas. Acesso em 29 de agosto, 2019. Disponível:

https://nacoesunidas.org .

Oliveira Leite, L. \& Rezende, D. A. (2017). E-gov. estratégico: governo eletrônico para gestão do desempenho da administração pública. Appris Editora e Livraria Eireli-ME,

Portal da Prefeitura Municipal de Bombinhas. Acesso de 04 de abril, 2017 a 08 de junho, 2018. Disponível: http://www.bombinhas.sc.gov.br . 
Portal da Prefeitura Municipal de Ilhabela. Acesso de 15 de junho de 2017 à 08 de junho de 2018. Disponível: http://www.ilhabela.sp.gov.br.

Programa Cidades Sustentáveis. Acesso em 18 de maio de 2018. Disponível: http://www.cidadessustentaveis.org.br,

REGO, J. A. A. et al. (2013). Cidades Sustentáveis: Lidando com a urbanização de forma ambiental, social e economicamente sustentável.

Sachs, I. (2007) Organizado por Nascimento, E. P. \& Vianna. J. N. Dilemas e desafios do desenvolvimento sustentável no Brasil, Rio de Janeiro. Garamond.

Sachs, I. (2009). Caminhos para o desenvolvimento sustentável. Rio de Janeiro. Garamond.

SAMPIERI, Roberto Hernandez; COLLADO, Carlos Fernández; BAPTISTA LUCIO, Maria del Pilar. (2013). Metodologia de pesquisa. 5. Ed. Porto Alegre. Penso.

Sen, A. (2010). Desenvolvimento como liberdade; tradução Motta, L. T. São Paulo: Companhia das Letras.

SNIS - Sistema Nacional de Informações sobre Saneamento. Acesso em 11 de novembro, 2017. Disponível: http://www.snis.gov.br

YIN, Robert K. (2015). Estudo de caso: planejamento e métodos. 5.ed. Porto Alegra. Bookman.

Recebido: 09 dez. 2019

Aprovado: $11 \mathrm{fev} .2020$

DOI: $10.3895 /$ rbpd.v9n1.10684

Como citar: FRANZ, N. M.; ANDREOLI, C. V.; SILVA, C. L. Políticas públicas e o desenvolvimento sustentável de cidades litorâneas: estudo de caso Bombinhas (SC) e llhabela (SP). R. bras. Planej. Desenv. Curitiba, v. 9, n. 1, p. 60-71, jan./abr. 2020. Disponível em: <https://periodicos.utfpr.edu.br/rbpd>. Acesso em XXX.

Correspondência:

Nádia Mara Franz

Av. Sete de Setembro, 3165 - Rebouças - Curitiba, PR

Direito autoral: Este artigo está licenciado sob os termos da Licença CreativeCommons-Atribuição 4.0

Internacional. 